\title{
Chronic High-Fat Diet Drives Postnatal Epigenetic Regulation of $\mu$-Opioid Receptor in the Brain
}

\author{
Zivjena Vucetic', Jessica Kimmel' and Teresa M Reyes*,', \\ 'Institute for Translational Medicine and Therapeutics, Department of Pharmacology University of Pennsylvania, School of Medicine, Philadelphia, \\ PA, USA
}

\begin{abstract}
Opioid system dysregulation has been observed in both genetic and high-fat diet (HFD)-induced models of obesity. An understanding of the molecular mechanisms of MOR transcriptional regulation, particularly within an in vivo context, is lacking. Using a diet-induced model of obesity (DIO), mice were fed a high-fat diet (60\% calories from fat) from weaning to $>18$ weeks of age. Compared with mice fed the control diet, DIO mice had a decreased preference for sucrose. MOR mRNA expression was decreased in reward-related circuitry (ventral tegmental area (VTA), nucleus accumbens (NAc), and prefrontal cortex (PFC)) but not the hypothalamus, important in the homeostatic regulation of feeding. DNA methylation is an epigenetic modification that links environmental exposures to altered gene expression. We found a significant increase in DNA methylation in the MOR promoter region within the reward-related brain regions. Methyl CpG-binding protein 2 (MeCP2) can bind methylated DNA and repress transcription, and DIO mice showed increased binding of MeCP2 to the MOR promoter in reward-related regions of the brain. Finally, using ChIP assays we examined H3K9 methylation (inactive chromatin) and $\mathrm{H} 3$ acetylation (active chromatin) within the MOR promoter region and found increased $\mathrm{H} 3 \mathrm{~K} 9$ methylation and decreased $\mathrm{H} 3$ acetylation. These data are the first to identify DNA methylation, MeCP2 recruitment, and chromatin remodeling as mechanisms leading to transcriptional repression of MOR in the brains of mice fed a high-fat diet.

Neuropsychopharmacology (201 I) 36, I199-1206; doi:I0.1038/npp.20I I.4; published online I6 February 201 I
\end{abstract}

Keywords: $\mu$-opioid receptor; preproenkephalin; DNA methylation; MeCP2; obesity; reward

\section{INTRODUCTION}

The $\mu$-opioid receptor (MOR) is expressed broadly throughout the central nervous system (CNS) and participates in a wide array of physiological functions. Notably, MOR has a central role in coding the rewarding properties of natural stimuli such as palatable foods, as well as opiate drugs (eg, morphine, and heroin (Klein et al, 2009)) and nicotine (Berrendero et al, 2010) (also, see excellent, extensive recent review (Merrer et al, 2009)).

Little is known about MOR expression regulation in the brain. MOR expression can be altered by dopaminergic drugs, such as cocaine (Leri et al, 2006), whereas chronic exposure to opioid agonists or antagonists does not alter mRNA expression (physiological responses, such as tolerance and dependence are thought to involve posttranscriptional modifications that affect MOR function (Ammon-Treiber and Höllt, 2005)). Importantly, changes

*Correspondence: Dr TM Reyes, Department of Pharmacology, University of Pennsylvania School of Medicine, 805 Biomedical Research Building II/III, 42I Curie Boulevard, Philadelphia, PA 191046160, USA, Tel: +215 573 2991, Fax: + 2155739004 ,

E-mail: reyestm@mail.med.upenn.edu

Received 13 December 2010; revised 4 January 2011; accepted 5 January 201 I in diet and/or metabolic state have been shown to influence expression of MOR. Hypothalamic MOR mRNA expression was increased following a 48-h fast (Barnes et al, 2008), whereas chronic exposure to a high-fat diet (HFD) can decrease MOR mRNA in the ventral tegmental area (VTA) (Blendy et al, 2005). Additionally, increased MOR binding has been shown in certain brain regions in the rat in response to diet-induced obesity (DIO) (Smith et al, 2002).

Epigenetic mechanisms of gene regulation, such as DNA methylation and histone modifications (eg, methylation, acetylation, ubiquitination) can regulate gene expression in response to environmental stimuli, such as drugs of abuse or diet. Recent reports have identified epigenetic modifications in the CNS in response to altered diet, particularly in the prenatal or early postnatal time period, when brain development is particularly vulnerable to perturbations (Vucetic et al, 2010a,b). Epigenetic regulation of gene expression outside of this early vulnerable time period has been documented as well, in response to cocaine (Schroeder et al, 2008), stress (Renthal et al, 2007), and learning and memory (Miller et al, 2010). Given that MOR expression can be affected by HF diet, the goal of these studies was to determine whether epigenetic modifications of the MOR occur during the postnatal period in response to chronic intake of HFD. 


\section{MATERIALS AND METHODS}

\section{Animals and Experimental Model}

C57BL/6J females were bred to DBA/2J males. During pregnancy and lactation, females were fed a controlformulated diet (Test Diet, Richmond, IN) (\#5755) that contained $18.5 \%$ protein, $12 \%$ fat, and $69.5 \%$ carbohydrate. At weaning, half the pups were placed on a HFD (\#58G9; $18.5 \%$ protein, $60 \%$ fat, and $20.5 \%$ carbohydrate) and half continued on the control diet. Litter size was six to eight pups (one to two pups were culled when necessary). One animal per litter from 10-15 different dams was used in individual experiments, to control for any litter effect. Body weights were recorded weekly, and male mice $(n=$ 5-8/group) aged 18-24 weeks of age were used in all experiments. All procedures were approved by the Institutional Animal Care and Use Committee (IACUC) of the University of Pennsylvania.

\section{Sucrose Preference}

For the duration of the test, offspring were caged individually and fed ad libitum. Mice were maintained on their respective diets (control or high fat) during testing, as removal of a palatable diet is a stressor (Pankevich et al, 2010). Mice were given simultaneous access to two bottles, one with water and one with $4 \%$ sucrose solution for $48 \mathrm{~h}$. Bottle order was random and was switched after $24 \mathrm{~h}$. Intake of consumed water, sucrose solution, and animal weight were measured every $24 \mathrm{~h}$. Water, sucrose, and the total fluid intake were normalized to body weight. Sucrose preference was calculated as a percentage of sucrose solution consumed in relation to total fluid intake.

\section{Genomic DNA and Total RNA Isolation from Brain}

Animals were euthanized with an overdose of carbon dioxide, followed by cervical dislocation, a method consistent with the recommendations of the Panel on Euthanasia of the American Veterinary Medical Association. After animals were killed, brains were rapidly removed and placed in RNA later (Ambion, Austin, TX) for $4 \mathrm{~h}$ before dissections. Brain dissections were performed as previously described (Cleck et al, 2008; Reyes et al, 2003; Vucetic et al, 2010a) and as detailed in Supplementary Methods. Genomic DNA (gDNA) and total RNA were isolated simultaneously using AllPrep DNA/RNA Mini Kit (Qiagen).

\section{Gene Expression Analysis by Quantitative Real-Time PCR}

For each individual sample, $500 \mathrm{ng}$ of total RNA was used in reverse transcription using High Capacity Reverse Transcription Kit (ABI, Foster City, CA). Expression of target genes was determined by quantitative RT-PCR using genespecific TaqMan probes (ABI) with TaqMan Gene Expression Master Mix (ABI) on the ABI7900HT Real-Time PCR Cycler. Probes used for RT-PCR are listed in Supplementary Material (Table S1). The relative amount of each transcript was determined using delta Ct values as previously described (Pfaffl, 2001). Changes in gene expression were calculated against endogenous, unchanged $\beta$-actin standard.

\section{Methylated DNA Immunoprecipitation (MeDIP) Assay}

Methylated DNA Immunoprecipitation (MeDIP) assay was performed as described (Weber et al, 2005). Methylated DNA was immunoprecipitated using $10 \mu \mathrm{g}$ of mouse monoclonal 5-methylcytidine antibody (Eurogentec) or mouse pre-immune serum. Enrichment in the MeDIP fraction was determined by quantitative RT-PCR using chromatin immunoprecipitation (ChIP)-qPCR Assay Master Mix (SuperArray) on the ABI7900HT Real-Time PCR Cycler. For all genes examined, primers were obtained from Superarray (ChIP-qPCR Assays (-01) kb tile, SuperArray) for the amplification of genomic regions spanning the CpG sites located 300-500-bp upstream of the transcription start sites (see Supplementary Material for primer sequences, Table S2). MeDIP results were expressed as fold enrichment of immunoprecipitated DNA for each specific site. To calculate differential occupancy fold change (\% enrichment), the MeDIP DNA fractions' Ct values were normalized to the Input DNA fraction $\mathrm{Ct}$ value (see Supplementary Material). Finally, the normalized level of DNA methylation at a particular site was expressed as relative to control group set to 1 .

\section{ChIP Assays}

Chromatin was prepared from dissected brain regions as previously described (Chahrour et al, 2008). ChIP-IT express (Active Motif) kit with enzymatic shearing protocol was used for ChIP assays according to manufacturer's instructions. Immunoprecipitations were performed using $5 \mathrm{mg}$ of the following antibodies: normal serum IgG (Millipore), anti-di-methyl histone H3-K9 (Millipore), anti-acetyl histone $\mathrm{H} 3$ (Millipore), and anti-methyl CpGbinding protein 2 (MeCP2) (Millipore). Enrichment of genomic DNA was assessed using $\mathrm{qPCR}$ as described for MeDIP.

\section{Statistical Analyses}

Data, presented as means \pm SEM, were analyzed using Prism 4 (GraphPad) and Excel tools for statistical analysis. Student's $t$-test was used to analyze differences in sucrose preference and gene expression between DIO and control animals, with Bonferroni's correction for multiple $t$-tests (multiple genes within a brain region) applied as warranted. A $p$-value of 0.05 or lower was considered significant.

\section{RESULTS}

Mice had continuous access to control diet (CD) or $60 \%$ HFD diet for 15-17 weeks (from weaning at 3 weeks until 18-20 weeks of age). At 20 weeks of age, HFD-induced obese (DIO) animals showed on average $27 \%$ greater body weight compared with controls (Figure 1a, $t$-test $p<0.05$ ). Twobottle sucrose preference test ( $4 \%$ sucrose) was performed to assess the response of control and DIO mice to a naturally rewarding stimulus. Although both groups of animals showed a preference for sucrose over water, DIO mice displayed a significantly reduced preference for the sucrose solution compared with control animals (Figure $1 \mathrm{~b}$, $t$-test, $p<0.05$ ), suggesting a state of reward hypofunction in the DIO animals. 
a

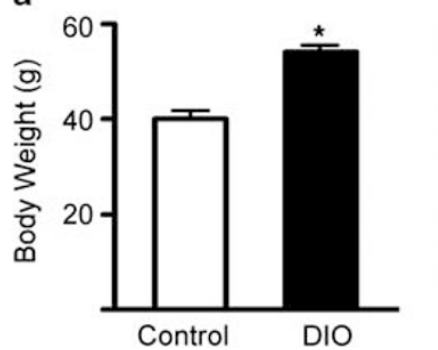

b

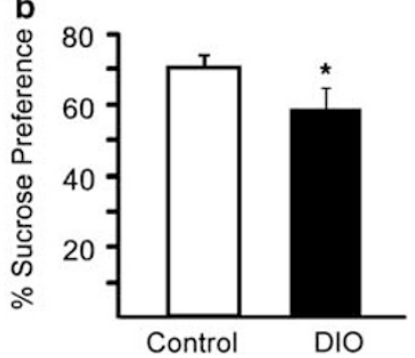

Figure I Long-term exposure to high-fat diet (HFD) induces obesity in mice, and decreases preference for sucrose. Mice had continuous access to control or 60\% HFD from weaning at 3 weeks until 18-20 weeks of age. (a) Dietary-induced obese mice (DIO) were $27 \%$ heavier than controls at 20 weeks. (b) Preference for $4 \%$ sucrose solution was evaluated using 48-h two-bottle preference test in control mice (white bar) and DIO mice (black bar). DIO mice showed a decreased preference for sucrose compared with control-diet fed animals. Values represent mean \pm SEM. $* p<0.05, n=6-8 /$ group.
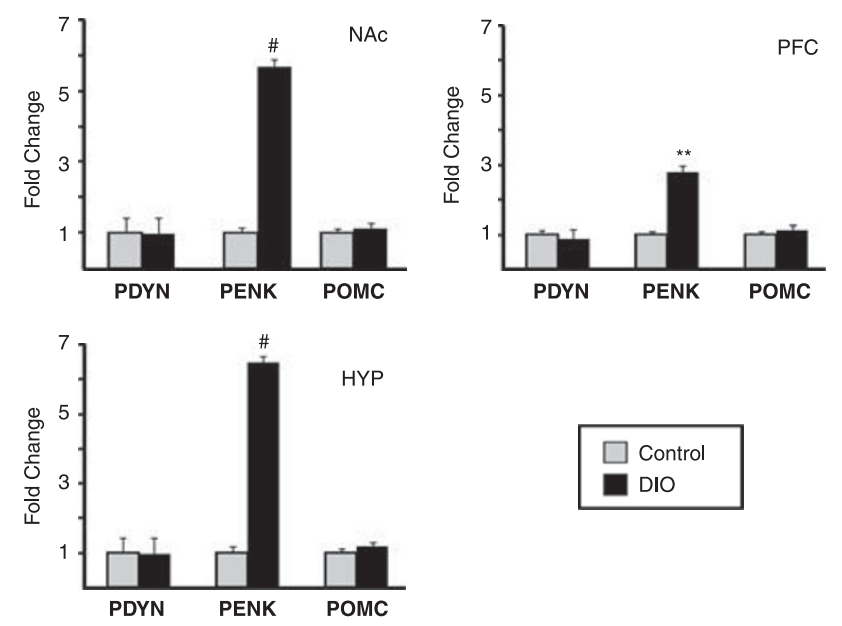

Figure 2 Expression of endogenous opioid ligand precursors in control and obese mice. RT-qPCR was used to assay mRNA levels of prodynorphin (PDYN), preproenkephalin (PENK) and pro-opiomelanocortin (POMC) mRNA in nucleus accumbens (NAc), prefrontal cortex (PFC), and HYP of control (gray bars) and obese mice (Dietary-induced obese mice (DIO), black bars). PENK levels were significantly increased in DIO mice in all CNS regions tested. Mean \pm SEM. ${ }^{*} * p<0.01,{ }^{\#} p<0.005$.

To examine whether chronic HFD affected opioid expression within structures of the reward circuitry (VTA, nucleus accumbens (NAc), prefrontal cortex (PFC)) or hypothalamus, RT-qPCR was used to measure mRNA levels of the endogenous ligand precursors, prodynorphin (PDYN), preproenkephalin (PENK), and pro-opiomelancortin (POMC). Expression of PENK mRNA was robustly increased (three to six fold) in the NAc, PFC and HYP of DIO animals ( $p<0.005, p<0.01$ and $p<0.005$, respectively), whereas levels of preprodynorphin and pro-opiomelanocortin remained unchanged (Figure 2). In addition to the opioid ligands, mRNA for the receptors was also examined. Expression of MOR mRNA was significantly decreased in areas of the brain related to reward processing (NAc, PFC, VTA) in DIO animals $(p<0.01, p<0.005$ and $p<0.005$, respectively), whereas remaining unchanged in the hypothalamus (Figure 3). Further, in the VTA, expression of
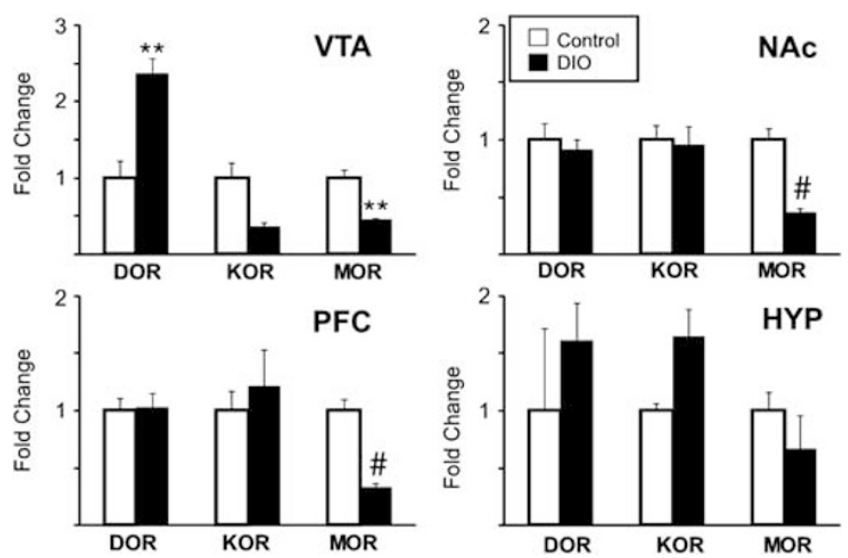

Figure 3 Expression of opioid receptors in control and obese mice. RT-qPCR was used to assay mRNA levels of delta (DOR), kappa (KOR) and mu-opioid (MOR) receptors in ventral tegmental area (VTA), nucleus accumbens (NAc), prefrontal cortex (PFC) and HYP of control (Control; gray bars) and obese mice (Dietary-induced obese mice (DIO), black bars). DOR was significantly increased in VTA, whereas MOR levels were significantly reduced in VTA, PFC and NAc of DIO mice. KOR levels displayed a trend toward decreased levels in the VTA $(p<0.05$, Bonferroni corrected $p$-value $=0.0165)$ Values are mean \pm SEM. *** $p<0.01$, ${ }^{*} p<0.005$.

kappa opioid receptor was decreased $(p<0.05$, although not significant at Bonferroni corrected $p=0.05 / 3=.016$ ), whereas expression of mRNA for the delta subtype of the opioid receptor was significantly increased $(p<0.01)$.

Given the consistent downregulation of the MOR mRNA expression within the reward circuitry and the importance of MOR for coding reward, further experiments were designed to examine in vivo epigenetic modifications related to these expression differences. The promoter region of the MOR was found to be significantly hypermethylated in the VTA, NAc, and PFC of DIO animals, brain regions in which mRNA expression was decreased (Figure 4). As expected, methylation of the MOR promoter did not differ in samples taken from the hypothalamus. DNA methylation within the promoter region of PENK was also examined and did not differ between groups in any brain region (data not shown).

$\mathrm{MeCP} 2$ can function as a transcriptional repressor and has been reported to interact with the MOR receptor (Hwang et al, 2007, 2009), so ChIP assays were completed to determine whether MeCP2 was differentially bound to the MOR promoter in the CNS samples from DIO animals. Binding of MeCP2 to a region of the MOR promoter $0.5-\mathrm{kb}$ upstream of the transcriptional start site was significantly increased in the NAc and PFC of DIO animals, and significantly decreased in the hypothalamus (Figure 5). Importantly, MeCP2 binding in regions of the MOR not known to be involved in transcriptional control, either 2-kb upstream or $0.5-\mathrm{kb}$ downstream of the start site, were not differentially bound by $\mathrm{MeCP} 2$, nor was there differential binding to GAPDH. Next, ChIP was used to examine the association of additional epigenetic marks, associated with either inactive chromatin (H3-K9 dimethylation, (Snowden et al, 2002)) or active chromatin (H3 acetylation, Lee et al, 1993), with the MOR promoter region. There was an increase in $\mathrm{H} 3-\mathrm{K} 9$ methylation associated with the MOR 


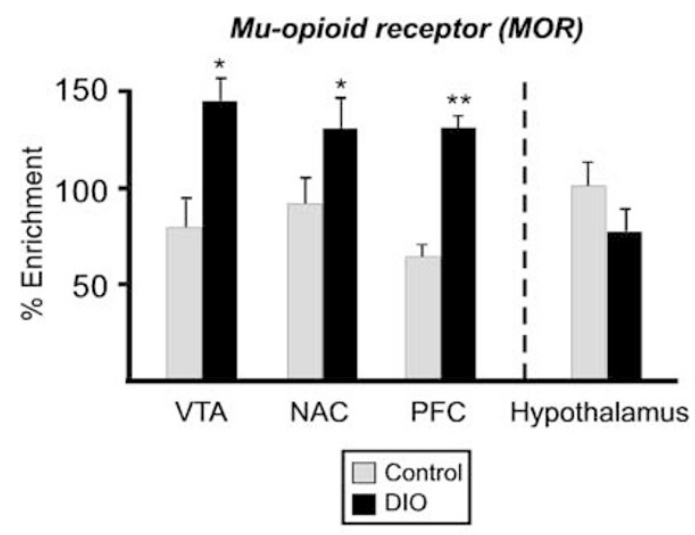

Gapdh

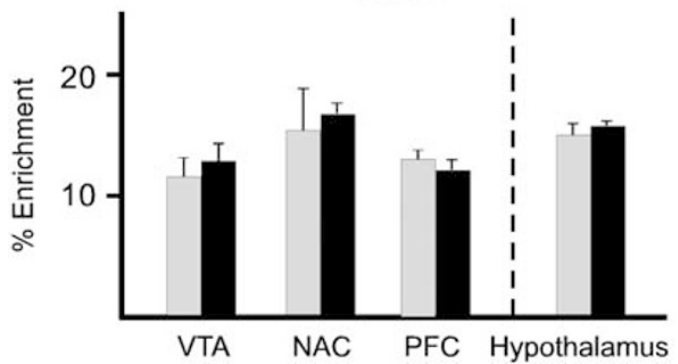

Figure 4 DNA methylation status of $\mu$-opioid receptor (MOR) promoter in dietary-induced obese mice (DIO) mice. Genomic DNA was isolated from dissected ventral tegmental area (VTA), nucleus accumbens (NAc), prefrontal cortex (PFC), and HYP of control (gray bars) and obese (DIO) mice, sheared by sonication and immunoprecipitated with 5-methylcytosine antibody. The enrichment of DNA methylation relative to input genomic DNA at the proximal promoter region of MOR was quantified by qPCR. DIO mice displayed hypermethylation of MOR. GAPDH methylation was not altered in DIO mice. Values are mean \pm SEM. $* p<0.05$, *** $p<0.0$ I, $n=6 /$ group, Two-tailed $t$-test. receptor promoter in the VTA, NAc, and PFC of DIO animals, whereas there was a decrease in $\mathrm{H} 3$ acetylation associated with the MOR in the NAc and PFC from the DIO animals (Figure 6). Neither of the histone modifications was differentially associated with MOR in the hypothalamus.

\section{DISCUSSION}

These experiments provide important new data describing epigenetic alterations linking chronic consumption of HFD with MOR gene repression in the CNS. We found that a decrease in $M O R$ gene expression within reward-related circuitry (NAc, PFC and VTA) was associated with epigenetic changes suggestive of a repressed transcriptional state at the histone and DNA level (DNA hypermethylation within the promoter region of $M O R$, increased binding of $\mathrm{MeCP} 2$ within the active promoter region of the MOR, an increase in $\mathrm{H} 3-\mathrm{K} 9$ methylation and a decrease in $\mathrm{H} 3$ acetylation). Observations within the VTA were consistent, but more variable, such that increased $\mathrm{MeCP} 2$ binding to the MOR promoter and decreased $\mathrm{H} 3$ acetylation were not significantly different. Decreased MeCP2 binding to the MOR promoter was observed in the hypothalamus; however, this did not lead to altered MOR expression.

Chronic consumption of HFD can result in a state of reward hypofunction ((Cottone et al, 2009; Johnson and Kenny, 2010; Stice et al, 2008) and supported here in the observed decrease in sucrose preference). It has been suggested that reduced function of the reward system can increase motivation to consume palatable foods or seek drugs of abuse in an effort to mitigate the 'state' of reduced reward (Johnson and Kenny, 2010; Koob and le Moal, 2008). Reward hypofunction in obesity has been linked to
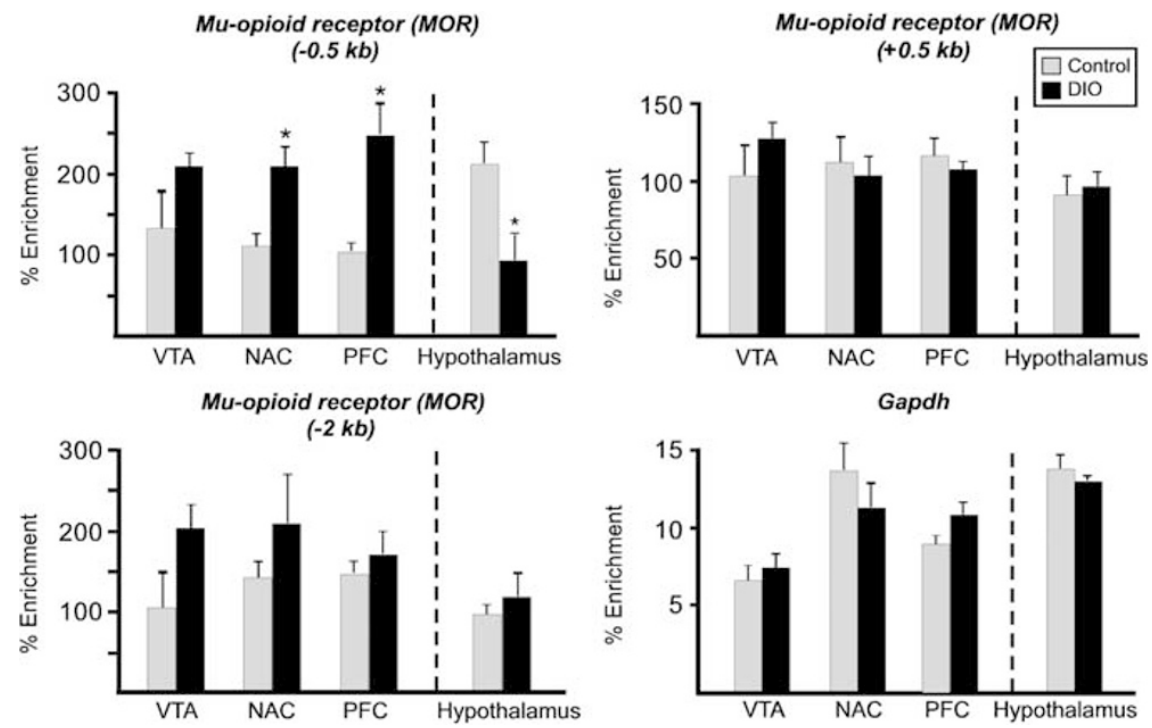

Figure 5 Association of methyl CpG-binding protein 2 (MeCP2) on $\mu$-opioid receptor (MOR) promoter in central nervous system (CNS) of dietaryinduced obese mice (DIO) mice. Chromatin was isolated from dissected ventral tegmental area (VTA), nucleus accumbens (NAc), prefrontal cortex (PFC), and HYP of control (gray bars) and DIO mice (black bars), enzymatically digested and immunoprecipitated with lgG and anti- MeCP2. The enrichment of genomic DNA associated with immunoprecipitated chromatin around proximal promoter $(-0.5$ and $+0.5 \mathrm{~kb})$ and $2 \mathrm{~kb}$ distal of MOR was quantified by qPCR. DIO mice displayed increased association of MeCP2 only in the $-0.5-k b$ region of the MOR promoter in NAc and PFC, and decreased MeCP2 association in the hypothalamus. There was no difference in MeCP2 binding either +0.5 or $-2.0 \mathrm{~kb}$ from the MOR promoter and no differential binding to GAPDH. Values are mean \pm SEM. $* p<0.05, n=3$ /group, Two-tailed $t$-test. 
Mu-opioid receptor (MOR)

H3-K9 methylation Inactive Chromatin

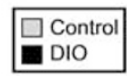

H3 acetylation

Active Chromatin
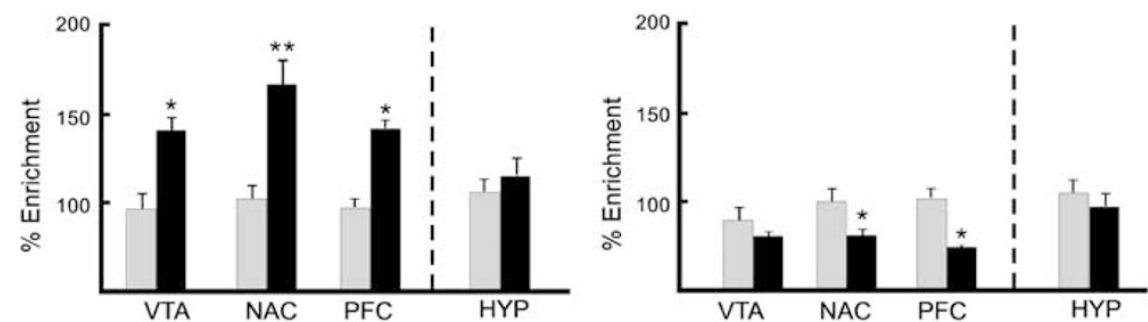

Figure 6 Histone modifications on $\mu$-opioid receptor (MOR) promoter in central nervous system (CNS) of dietary-induced obese mice (DIO) mice. Chromatin was isolated from dissected ventral tegmental area (VTA), nucleus accumbens (NAc), prefrontal cortex (PFC), and HYP of control (gray bars) and DIO mice (black bars), enzymatically digested and immunoprecipitated with IgG, anti di-methyl-H3K9 (inactive chromatin) and anti-acetyl-H3 antibody (active chromatin). The enrichment of genomic DNA associated with immunoprecipitated chromatin at proximal promoter regions of the MOR was quantified by qPCR as described for MeDIP. DIO mice displayed increased amount of methylated histone on the MOR promoter, whereas the amount of acetyl-histone associated DNA was reduced in NAc and PFC of DIO mice. Changes in histone modifications were not observed on GAPDH promoter in $\mathrm{DIO}$ mice. This suggests an epigeneticlly repressed state of the MOR promoter in DIO mice. Values are mean $\pm \mathrm{SEM}$. $* p<0.05, * * 2<0.0 \mathrm{I}, n=6 / \mathrm{group}$, Two-tailed t-test.

decreased dopamine $\mathrm{D} 2$ receptor expression and function in both animal models of obesity, as well as in humans (Johnson and Kenny, 2010; Stice et al, 2008; Wang et al, 2001). Further, obese rats were shown to have decreased basal levels of dopamine in the nucleus accumbens, as well as attenuated dopamine release in response to chow or amphetamine (Geiger et al, 2009). Additionally, reward hypofunction can result from repeated cycling between a highly palatable and a chow diet, and the CRF system has been implicated in this response (Cottone et al, 2009). These data identify downregulation of the MOR as an additional mechanism that may contribute to a diminished state of reward in obese animals. Similar findings, including decreased MOR expression in the VTA and diminished nicotine-related reward behavior, were reported in a different mouse strain (129SvEv/C57BL/6) (Blendy et al, 2005) and complement the present findings.

A limitation of the current experiments is the fact that concurrent consumption of calorically dense HFD in the DIO mice could contribute to the decreased sucrose preference in DIO mice. And in fact, consumption of a HFD in the absence of obesity has been shown to attenuate responding for sucrose in an operant task (Davis et al, 2008). However, in this same report, the authors also showed that obesity, independent of consumption of the HFD, also reduced responding for sucrose and eliminated the development of a conditioned place preference for amphetamine. In contrast, in a separate study that included a pair-fed cohort, deficits in dopamine function in obesity were linked specifically to consumption of a HFD, not weight gain, per se (Alsiö et al, 2010). Collectively, these data support the finding of reward hypofunction in obesity and also identify a complex interplay of diet, metabolic state and reward-related behaviors.

The increased expression of PENK was also notable, as increased PENK mRNA, particularly in the NAc (Ellgren et al, 2007), may be related to drug abuse vulnerability (Nikoshkov et al, 2008). Further, increased PENK expression may potentiate the obesity cycle, as injections of enkephalin peptides stimulate consumption of fat-rich diets, particularly that of a HFD over a low-fat diet and a fat-rich diet over a carbohydrate-rich diet (Leibowitz, 2007). Additionally, increased expression of the delta opioid receptor $(D O R)$ in the VTA was observed. Although the functional relevance of this increase is unclear at the present, inactivation of the DOR in the VTA increased the rewarding properties of cocaine (Ward and Roberts, 2007), suggesting that increased DOR in the VTA may also participate in establishing reward hypofunction. Given the focus of this manuscript on transcriptional regulation, the current studies examined gene expression at the mRNA level and did not examine protein expression. However, our results closely parallel findings from a similar study that determined the concentration of binding sites for the opiate receptor subtypes in lean and genetically obese mice (Khawaja et al, 1989), in which obese mice demonstrated an increase in DOR binding, a decrease in MOR binding and no change in KOR binding. Collectively, these data support the idea that chronic consumption of a HFD leads to opioid dysfunction within reward circuitry.

Altered DNA methylation in the brain in response to adverse conditions, either in utero or in the early postnatal timeperiod, has been documented across multiple challenge conditions (eg, altered diet or prenatal stress) (Coupé et al, 2010; Murgatroyd et al, 2009; Niculescu et al, 2006; Niculescu and Lupu, 2009; Plagemann et al, 2009; Vucetic et al, 2010a, b; Weaver et al, 2004). Given the relative decrease in CNS plasticity across developmental time, the question of whether altered DNA methylation occurs in the adult brain remains an important issue, and one that has been recently addressed. Differential methylation of $B D N F$ during memory consolidation (Lubin et al, 2008), and altered DNA methylation of $F k b p 5$ in response to chronic corticosterone exposure (Lee et al, 2010b) has been observed. Further, the importance of balanced DNA methylation for behavioral responses to chronic cocaine or chronic social defeat has recently been reported (LaPlant et al, 2010). To the best of our knowledge, only one other paper has reported differential DNA methylation in DIO mice (hypomethylation of melanocortin 4 receptor in whole 
brain) (Widiker et al, 2010). The current manuscript significantly extends this work by examining DNA methylation of $M O R$ and PENK across multiple brain regions.

These findings have important implications for reward circuitry function in obesity, as gene expression changes driven by differential DNA methylation may prove relatively more stable. Although removal of the HFD and/or normalization of body weight was not examined in the present experiments, related data in the literature suggest the relative stability of reward-related changes in obesity. Decreased expression of the dopamine D1 receptor in the accumbens of obese rats persisted 18 days after the withdrawal of the HFD (Alsiö et al, 2010) and increased brain reward thresholds in obesity persisted 2 weeks after the removal of a HFD, even as body weight was normalized (Johnson and Kenny, 2010). Additionally, it should be noted that in these studies high fat diet consumption began when animals were 3 weeks of age, approximately mid-adolescence, which is clearly a critical period in brain development (Ernst et al, 2009). Related experiments that have examined gene expression changes in the CNS in obesity have identified the length of diet exposure and the timing of the exposure as critical variables (eg, early and chronic exposure to HFD altered CNS gene expression (Lee et al, 2010a), whereas 4-week HFD exposure in adult animals did not (de Leeuw van Weenen et al, 2009)). The present experiments were designed to cover the broadest possible range of postnatal exposure, and future experiments will be directed at defining the critical period of exposure for epigenetic alterations.

It was striking to note that decreased MOR expression and DNA hypermethylation were not universally observed throughout the brain, as we observed these changes within the reward circuitry but not within the hypothalamus. This suggests the existence of a mechanism whereby regional specificity is encoded. Given that the promoter sequence across these different regions does not differ, differential/ preferential activation of neurons within the reward circuitry during the chronic consumption of the HFD likely participates in driving some of the epigenetic modifications. This would be in line with similar observations linking acute neuronal activation to epigenetic modifications (Ma et al, 2009; Zhou et al, 2006). One possible contributor is the increase in MOR agonist stimulation (provided by increased PENK), as this has been shown to downregulate MOR mRNA expression in vitro (Gach et al, 2008). Additionally, there are other likely contributors to the initiation of the epigenetic changes that drive altered $M O R$ expression. Increased adiposity and elevated glucose and leptin are well-characterized responses in the DIO paradigm (Matysková et al, 2008). Further, pro-inflammatory cytokines are elevated in obesity, and IL-4 has been shown to modify epigenetic regulation of MOR expression in T-cells (Kraus et al, 2010). The direct contribution of the HFD in combination with the physiological responses to the diet are likely to have synergistic roles in driving changes in MOR expression.

Epigenetic control of $M O R$ expression during development involves altered MeCP2 binding and histone modifications, seen both in vitro (Hwang et al, 2007) and in vivo (Hwang et al, 2009). Histone modifications associated with MOR expression changes have been demonstrated in the hippocampus in response to ischemia (Formisano et al, 2007), and an excellent recent review (Wei and Loh, 2011) describes the current state of the literature regarding transcriptional regulation of the MOR. This report is the first to show both altered DNA methylation and chromatin changes around the MOR promoter in response to chronic HFD consumption. Defining whether these changes are reversed upon removal of the diet and/or normalization of body weight will have significant implications for understanding the way the CNS is altered in obesity.

The prevalence of obesity, at epidemic proportions and continuing to rise (Sherry et al, 2010), is driven by the chronic intake of highly palatable (high fat, high sugar), energy-dense foods (coupled with reduced activity levels). Maintenance of weight loss after dieting is typically low (with 67 (Phelan et al, 2010) to 80\% (Field et al, 2001) of patients regaining the lost weight). These data identify postnatal epigenetic regulation of MOR repression as a novel CNS response to the chronic intake of HFD; a response which may, among other things, contribute to the difficulty in establishing healthier eating patterns after chronic exposure to highly palatable foods.

\section{ACKNOWLEDGEMENTS}

We thank Kathy Totoki for technical assistance. The current work was supported by NIH DK064086 (Reyes) and MH091372 (Reyes).

\section{DISCLOSURE}

The authors declare no conflict of interest.

\section{REFERENCES}

Alsiö J, Olszewski PK, Norbäck AH, Gunnarsson ZE, Levine AS, Pickering $C$ et al (2010). Dopamine d1 receptor gene expression decreases in the nucleus accumbens upon long-term exposure to palatable food and differs depending on diet-induced obesity phenotype in rats. Neuroscience 171: 779-787.

Ammon-Treiber S, Höllt V (2005). Morphine-induced changes of gene expression in the brain. Addict Biol 10: 81-89.

Barnes MJ, Primeaux SD, Bray GA (2008). Food deprivation increases the mrna expression of micro-opioid receptors in the ventral medial hypothalamus and arcuate nucleus. Am J Physiol Regul Integr Comp Physiol 295: R1385-R1390.

Berrendero F, Robledo P, Trigo JM, Martín-García E, Maldonado R (2010). Neurobiological mechanisms involved in nicotine dependence and reward: Participation of the endogenous opioid system. Neurosci Biobehav Rev 35: 220-231.

Blendy JA, Strasser A, Walters CL, Perkins KA, Patterson F, Berkowitz $\mathrm{R}$ et al (2005). Reduced nicotine reward in obesity: cross-comparison in human and mouse. Psychopharmacology (Berl) 180: 306-315.

Chahrour M, Jung SY, Shaw C, Zhou X, Wong ST, Qin J et al (2008). Mecp2, a key contributor to neurological disease, activates and represses transcription. Science 320: 1224-1229.

Cleck JN, Ecke LE, Blendy JA (2008). Endocrine and gene expression changes following forced swim stress exposure during cocaine abstinence in mice. Psychopharmacology (Berl) 201: 15-28.

Cottone P, Sabino V, Roberto M, Bajo M, Pockros L, Frihauf JB et al (2009). Crf system recruitment mediates dark side of compulsive eating. Proc Natl Acad Sci USA 106: 20016-20020. 
Coupé B, Amarger V, Grit I, Benani A, Parnet P (2010). Nutritional programming affects hypothalamic organization and early response to leptin. Endocrinology 151: 702-713.

Davis JF, Tracy AL, Schurdak JD, Tschöp MH, Lipton JW, Clegg DJ et al (2008). Exposure to elevated levels of dietary fat attenuates psychostimulant reward and mesolimbic dopamine turnover in the rat. Behav Neurosci 122: 1257-1263.

de Leeuw van Weenen JE, Hu L, Zelm KJ-V, Vries MGd, Tamsma JT, Romijn JA et al (2009). Four weeks high fat feeding induces insulin resistance without affecting dopamine release or gene expression patterns in the hypothalamus of c57bl6 mice. Brain Res 1250: 141-148.

Ellgren M, Spano SM, Hurd YL (2007). Adolescent cannabis exposure alters opiate intake and opioid limbic neuronal populations in adult rats. Neuropsychopharmacology 32: 607-615.

Ernst M, Romeo RD, Andersen SL (2009). Neurobiology of the development of motivated behaviors in adolescence: a window into a neural systems model. Pharmacol Biochem Behav 93: 199-211.

Field AE, Wing RR, Manson JE, Spiegelman DL, Willett WC (2001). Relationship of a large weight loss to long-term weight change among young and middle-aged us women. Int J obes Relat Metab Disord 25: 1113-1121.

Formisano L, Noh KM, Miyawaki T, Mashiko T, Bennett MV, Zukin RS (2007). Ischemic insults promote epigenetic reprogramming of mu opioid receptor expression in hippocampal neurons. Proc Natl Acad Sci USA 104: 4170-4175.

Gach K, Piestrzeniewicz M, Fichna J, Stefanska B, Szemraj J, Janecka A (2008). Opioid-induced regulation of mu-opioid receptor gene expression in the mcf-7 breast cancer cell line. Biochem Cell Biol 86: 217-226.

Geiger BM, Haburcak M, Avena NM, Moyer MC, Hoebel BG, Pothos EN (2009). Deficits of mesolimbic dopamine neurotransmission in rat dietary obesity. Neuroscience 159: 1193-1199.

Hwang CK, Song KY, Kim CS, Choi HS, Guo XH, Law PY et al (2007). Evidence of endogenous mu opioid receptor regulation by epigenetic control of the promoters. Mol Cell Biol 27: $4720-4736$.

Hwang CK, Song KY, Kim CS, Choi HS, Guo XH, Law PY et al (2009). Epigenetic programming of mu-opioid receptor gene in mouse brain is regulated by mecp2 and brg1 chromatin remodelling factor. J Cell Mol Med 13: 3591-3615.

Johnson PM, Kenny PJ (2010). Dopamine D2 receptors in addiction-like reward dysfunction and compulsive eating in obese rats. Nat Neurosci 13: 635-641.

Khawaja XZ, Bailey CJ, Green IC (1989). Central mu, delta, and kappa opioid binding sites, and brain and pituitary betaendorphin and met-enkephalin in genetically obese (ob/ob) and lean mice. Life Sci 44: 1097-1105.

Klein G, Rossi GC, Waxman AR, Arout C, Juni A, Inturrisi CE et al (2009). The contribution of mor-1 exons 1-4 to morphine and heroin analgesia and dependence. Neurosci Lett 457: 115-119.

Koob GF, le Moal M (2008). Addiction and the brain antireward system. Ann Rev Psychol 59: 29-53.

Kraus J, Lehmann L, Börner C, Höllt V (2010). Epigenetic mechanisms involved in the induction of the mu opioid receptor gene in jurkat $t$ cells in response to interleukin-4. Mol Immunol 48: $257-263$.

LaPlant Q, Vialou V, Covington HE, Dumitriu D, Feng J, Warren BL et al (2010). Dnmt3a regulates emotional behavior and spine plasticity in the nucleus accumbens. Nat Neurosci 13: 1137-1143.

Lee AK, Mojtahed-Jaberi M, Kyriakou T, Astarloa EA, Arno M, Marshall NJ et al (2010a). Effect of high-fat feeding on expression of genes controlling availability of dopamine in mouse hypothalamus. Nutrition 26: 411-422.

Lee DY, Hayes JJ, Pruss D, Wolff AP (1993). A positive role for histone acetylation in transcription factor access to nucleosomal DNA. Cell 72: 73-84.
Lee RS, Tamashiro KL, Yang X, Purcell RH, Harvey A, Willour VL et al (2010b). Chronic corticosterone exposure increases expression and decreases deoxyribonucleic acid methylation of fkbp5 in mice. Endocrinology 151: 4332-4343.

Leibowitz SF (2007). Overconsumption of dietary fat and alcohol: mechanisms involving lipids and hypothalamic peptides. Physiol Behav 91: 513-521.

Leri F, Zhou Y, Goddard B, Cummins E, Kreek MJ (2006). Effects of high-dose methadone maintenance on cocaine place conditioning, cocaine self-administration, and mu-opioid receptor mrna expression in the rat brain. Neuropsychopharmacology 31: $1462-1474$.

Lubin FD, Roth TL, Sweatt JD (2008). Epigenetic regulation of bdnf gene transcription in the consolidation of fear memory. J Neurosci 28: 10576-10586.

Ma DK, Jang MH, Guo JU, Kitabatake Y, Chang ML, PowAnpongkul N et al (2009). Neuronal activity-induced gadd45b promotes epigenetic DNA demethylation and adult neurogenesis. Science 323: 1074-1077.

Matysková R, Maletínská L, Maixnerová J, Pirník Z, Kiss A, Zelezná B (2008). Comparison of the obesity phenotypes related to monosodium glutamate effect on arcuate nucleus and/or the high fat diet feeding in c57bl/6 and nmri mice. Physiol Res 57: 727-734.

Merrer JL, Becker JA, Befort K, Kieffer BL (2009). Reward processing by the opioid system in the brain. Physiol Rev 89: 1379-1412.

Miller CA, Gavin CF, White JA, Parrish RR, Honasoge A, Yancey CR et al (2010). Cortical DNA methylation maintains remote memory. Nat Neurosci 13: 664-666.

Murgatroyd C, Patchev AV, Wu Y, Micale V, Bockmühl Y, Fischer D et al (2009). Dynamic DNA methylation programs persistent adverse effects of early-life stress. Nat Neurosci 12: 1559-1566.

Niculescu MD, Craciunescu CN, Zeisel SH (2006). Dietary choline deficiency alters global and gene-specific DNA methylation in the developing hippocampus of mouse fetal brains. FASEB J 20: 43-49.

Niculescu MD, Lupu DS (2009). High fat diet-induced maternal obesity alters fetal hippocampal development. Int J Dev Neurosci 27: 627-633.

Nikoshkov A, Drakenberg K, Wang X, Horvath MC, Keller E, Hurd YL (2008). Opioid neuropeptide genotypes in relation to heroin abuse: Dopamine tone contributes to reversed mesolimbic proenkephalin expression. Proc Natl Acad Sci USA 105: 786-791.

Pankevich DE, Teegarden SL, Hedin AD, Jensen CL, Bale TL (2010). Caloric restriction experience reprograms stress and orexigenic pathways and promotes binge eating. J Neurosci 30: 16399-16407.

Pfaffl MW (2001). A new mathematical model for relative quantification in real-time rt-pcr. Nucleic Acids Res 20: e45.

Phelan S, Wing RR, Loria CM, Kim Y, Lewis CE (2010). Prevalence and predictors of weight-loss maintenance in a biracial cohort results from the coronary artery risk development in young adults study. Am J Prev Med 39: 546-554.

Plagemann A, Harder T, Brunn M, Harder A, Roepke K, WittrockStaar M et al (2009). Hypothalamic proopiomelanocortin promoter methylation becomes altered by early overfeeding: An epigenetic model of obesity and the metabolic syndrome. J Physiol 587: 4963-4976.

Renthal W, Maze I, Krishnan V, Covington III HE, Xiao G, Kumar A et al (2007). Histone deacetylase 5 epigenetically controls behavioral adaptations to chronic emotional stimuli. Neuron 56: 517-529.

Reyes T, Walker JR, DeCino C, Hogenesch JB, Sawchenko PE (2003). Categorically distinct acute stressors elicit dissimilar transcriptional profiles in the paraventricular nucleus of the hypothalamus. J Neurosci 23: 5607-5616. 
Schroeder FA, Penta KL, Matevossian A, Jones SR, Konradi C, Tapper AR et al (2008). Drug-induced activation of dopamine $\mathrm{d}(1)$ receptor signaling and inhibition of class $\mathrm{i} / \mathrm{ii}$ histone deacetylase induce chromatin remodeling in reward circuitry and modulate cocaine-related behaviors. Neuropsychopharmacology 33: 2981-2992.

Sherry B, Blanck HM, Galuska DA, Pan L, Dietz WH, Balluz L (2010). Vital signs: state-specific obesity prevalence among adults - united states, 2009. MMWR 59: 1-5.

Smith SL, Harrold JA, Williams G (2002). Diet-induced obesity increases mu opioid receptor binding in specific regions of the rat brain. Brain Res 953: 215-222.

Snowden AW, Gregory PD, Case CC, Pabo CO (2002). Genespecific targeting of h3k9 methylation is sufficient for initiating repression in vivo. Curr Biol 12: 2159-2166.

Stice E, Spoor S, Bohon C, Small DM (2008). Relation between obesity and blunted striatal response to food is moderated by taqia al allele. Science 322: 449-452.

Vucetic Z, Kimmel J, Totoki K, Hollenbeck E, Reyes TM (2010a). Maternal high-fat diet alters methylation and gene expression of dopamine and opioid-related genes. Endocrinology 151: 47564764.

Vucetic Z, Totoki K, Schoch H, Whitaker KW, Hill-Smith T, Lucki I et al (2010b). Early life protein restriction alters dopamine circuitry. Neuroscience 168: 359-370.
Wang GJ, Volkow ND, Logan J, Pappas NR, Wong CT, Zhu W et al (2001). Brain dopamine and obesity. Lancet 357: 1883.

Ward SJ, Roberts DC (2007). Microinjection of the delta-opioid receptor selective antagonist naltrindole $5^{\prime}$-isothiocyanate site specifically affects cocaine self-administration in rats responding under a progressive ratio schedule of reinforcement. Behav Brain Res 182: 140-144.

Weaver I, Cervoni N, Champagne FA, D'Alessio AC, Sharma S, Seckl JR et al (2004). Epigenetic programming by maternal behavior. Nat Neurosci 7: 847-854.

Weber M, Davies JD, Wittig D, Oakeley EJ, Haase M, Wan L et al (2005). Chromosome-wide and promoter-specific analyses identify sites of differential DNA methylation in normal and transformed human cells. Nat Genet 37: 853-862.

Wei LN, Loh HH (2011). Transcriptional and epigenetic regulation of opioid receptor genes;present and future. Annu Rev Pharmacol Toxicol 51: 75-97.

Widiker S, Karst S, Wagener A, Brockmann GA (2010). High-fat diet leads to a decreased methylation of the mc4r gene in the obese bfmi and the lean b6 mouse lines. J Appl Genet 51: 193-197.

Zhou Z, Hong EJ, Cohen S, Zhao WN, Ho HY, Schmidt L et al (2006). Brain-specific phosphorylation of mecp2 regulates activity-dependent bdnf transcription, dendritic growth, and spine maturation. Neuron 52: 255-269.

Supplementary Information accompanies the paper on the Neuropsychopharmacology website (http://www.nature.com/npp) 